\title{
Random walks and diameter of finite scale-free networks
}

\author{
Sungmin Lee, Soon-Hyung Yook $*$ and Yup Kim $\dagger$ \\ Department of Physics and Research Institute for Basic Sciences, Kyung Hee University, Seoul 130-701, Korea
}

(Dated: October 4, 2018)

\begin{abstract}
Dynamical scalings for the end-to-end distance $R_{e e}$ and the number of distinct visited nodes $N_{v}$ of random walks (RWs) on finite scale-free networks (SFNs) are studied numerically. $\left\langle R_{e e}\right\rangle$ shows the dynamical scaling behavior $\left\langle R_{e e}(\bar{\ell}, t)\right\rangle=\bar{\ell}^{\alpha}(\gamma, N) g\left(t / \bar{\ell}^{z}\right)$, where $\bar{\ell}$ is the average minimum distance between all possible pairs of nodes in the network, $N$ is the number of nodes, $\gamma$ is the degree exponent of the SFN and $t$ is the step number of RWs. Especially, $\left\langle R_{e e}(\bar{\ell}, t)\right\rangle$ in the limit $t \rightarrow \infty$ satisfies the relation $\left\langle R_{e e}\right\rangle \sim \bar{\ell}^{\alpha} \sim d^{\alpha}$, where $d$ is the diameter of network with $d(\bar{\ell}) \simeq \ln N$ for $\gamma \geq 3$ or $d(\bar{\ell}) \simeq \ln \ln N$ for $\gamma<3$. Based on the scaling relation $\left\langle R_{e e}\right\rangle$, we also find that the scaling behavior of the diameter of networks can be measured very efficiently by using RWs.
\end{abstract}

PACS numbers: 89.75.Hc,05.40.Fb,89.75.Fb

For almost a decade there have been many studies on the topological properties of complex networks, since many structures of physically interacting systems are shown to form nontrivial complex structures [1, 2]. In these studies much effort has been put to investigate the physical origin of complex networks [3, 4]. It has been found that most of real web-like systems share several prominent structural features: scale-free degree distribution, high clustering, and small average path length (APL) [1, 3, 4, 5]. It is well known [6, 7] that random networks (RNs) [8] and small-world networks (SWNs) [9] have very small APL or diameter $d$ which scales as $d \sim \ln N$, where $N$ is the number of nodes. Recently, Cohen and Havlin [10] studied the diameter of scale-free networks (SFNs) [1] whose degree distribution $P(k)$ satisfies a power law $P(k) \sim k^{-\gamma}$. Using analytical arguments they showed [10] that

$$
d(N) \sim \begin{cases}\ln \ln N & , 2<\gamma<3 \\ \ln N / \ln \ln N & , \gamma=3 \\ \ln N & , \gamma>3 .\end{cases}
$$

For measuring the APL, the breath-first algorithm (BFA) [11] are mainly used. This algorithm is known to scale as $O\left(N^{2}\right)$. In this paper, we will show that random walk (RW) can provide more efficient method to find the scaling behavior of $d$ like Eq. (1).

The early studies on complex networks mainly focused on their topological properties. Recently, the physical systems whose elements interact along the links in complex networks have drawn much attention. Furthermore a number of studies have focused on the effects of the underlying topologies on the dynamical properties of such systems. Many dynamical systems on complex networks show rich behaviors which are far from the mean-field expectations and affected by the underlying topology [12, 13]. For example, the dynamical properties of RWs

*Electronic address: syook@khu.ac.kr

†Electronic address: ykim@khu.ac.kr on complex networks have been shown to be closely related to the topology of underlying networks [14, 15, 16]. Especially, the average number of distinct visited sites and the average end-to-end distance of RWs on SWNs are known to satisfy the scaling law [16]

$$
O(p, N, t)=O_{\text {sat }} F\left(p^{2} t, p N\right) .
$$

Here, $p$ is a density of shortcut and $O_{\text {sat }}$ is a saturated quantity.

It is well known that the SFNs have heterogeneous structures in which nodes with anomalously large number of degree and nodes with small degree co-exist [1, 2]. In SFNs, the dynamical properties of several systems are affected by the second moment of degree distribution $\left\langle k^{2}\right\rangle$ [17, 18, 19, 20]. It is interesting to study how the structural heterogeneity affects the scaling properties of RWs as changing the degree exponent $\gamma$. In this paper, we mainly investigate the dynamical scaling relation for the end-to-end distance $R_{e e}$ of RWs on finite SFNs with various $\gamma$. From the scaling relation, it will be shown that RWs on SFNs can provide much more efficient method to measure the scaling behavior of the diameter of finite SFNs than BFA. For the complementary purpose we also study the scaling relation for the number of distinct visited nodes $N_{v}$.

All the models are defined on a graph $G r=\{N, K\}$, where $N$ is the number of nodes in the network and $K$ is the total number of degrees with the average degree $\langle k\rangle=2 K / N$. Networks of the sizes $N=10^{3} \sim 10^{6}$ are used. To generate SFNs, we use the static network model introduced by Ref. 21] with $\langle k\rangle=4$. Initially a random walker is placed on a randomly chosen node denoted by $s$. At the time step $t+1$, the walker jumps to a randomly chosen node among the nearest neighbor nodes of the node where the walker is at $t$. The probability $P(i, t ; s, 0)$ to find the walker at node $i$ at $t$ thus follows the relation

$$
P(i, t+1 ; s, 0)=\sum_{j=1}^{N} \frac{A_{i j}}{k_{j}} P(j, t ; s, 0)
$$

with the condition $P(i, 0 ; s, 0)=\delta_{i, s}$ [15]. Here, $A_{i j}$ is the adjacency matrix whose elements are $A_{i j}=1(0)$ if two 
nodes $i$ and $j$ are connected (disconnected). All quantities are averaged over 100 network realizations and 1000 different initial positions of RWs for each network realization.

First, let us discuss the average end-to-end distance $\left\langle R_{e e}\right\rangle$ of RW on SFNs. At each time step $t$, we measure the shortest distance, $R_{e e}(t)$, from the node where the random walker is to the node $s$. By averaging $R_{e e}(t)$ over different initial positions and network realizations we get $\left\langle R_{e e}\right\rangle$. Here the shortest distance between two nodes in networks means the shortest path length or the minimal number of steps between them.

On a $D$-dimensional regular lattice, the average endto-end distance follows $\left\langle R_{e e}\right\rangle \sim t^{\nu}$ with $\nu=1 / 2[22$. However, on finite systems, $\left\langle R_{e e}\right\rangle$ saturates to a finite value in the limit $t \rightarrow \infty$. In this limit, the probability for each site to be occupied by a random walker is the same for all the sites and thus $\left\langle R_{e e}\right\rangle \sim \bar{\ell}$, where $\bar{\ell}$ is the minimum distance averaged over all possible pairs of sites [16]. On a SWN, for small $t\left(\ll 1 / p^{2}\right)$ regime $\left\langle R_{e e}\right\rangle$ again satisfies the same relation as on the regular lattice $\left(\left\langle R_{e e}\right\rangle \sim t^{1 / 2}\right)$, because the random walker cannot actually see shortcuts. But in the large $t$ regime $(t \gg$ $\left.\sqrt{\bar{\ell}^{2}} / p\right),\left\langle R_{e e}\right\rangle \sim \bar{\ell}$. For the intermediate time region $1 / p^{2} \ll t \ll \sqrt{\ell^{2}} / p$, Almaas et al. expected that $\left\langle R_{e e}\right\rangle \sim$ $\bar{\ell}$ satisfies the relation $\left\langle R_{e e}\right\rangle \sim t p[16]$.

A typical dependence of $\left\langle R_{e e}\right\rangle$ on $t$ for finite SFNs is shown in Fig. 1. Figure 1 shows the measured $\left\langle R_{e e}\right\rangle$

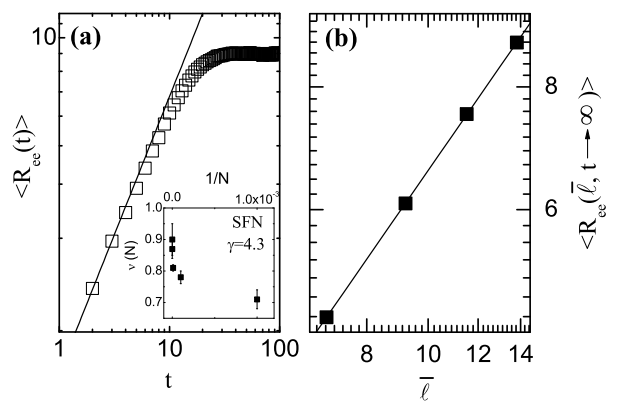

FIG. 1: Average end-to-end distance, $\left\langle R_{e e}\right\rangle$ on SFNs with $\gamma=2.4$ for $N=10^{7}$. (a) Plot of $\left\langle R_{e e}\right\rangle$ against $t$ in log$\log$ scale. The solid line correspond to $\nu=0.90$. The inset displays the dependence of the measured $\nu$ on $1 / N$. From the inset, we expect that $\nu \rightarrow 1$ in the limit $N \rightarrow \infty$. (b) Plot of $\left\langle R_{e e}(\bar{\ell}, t \rightarrow \infty)\right\rangle$ against $\bar{\ell}$ in $\log$-log scale with $\bar{\ell} \sim \ln N$. The solid line corresponds to $\alpha=0.93$.

on SFNs with $\gamma=4.3$ for $N=10^{7}$. From the early- $t$ behavior of $\left\langle R_{e e}\right\rangle$ (or the data for $t \lesssim 10$ in Fig. 1(a)) the obtained value of the exponent $\nu$ for the relation

$$
\left\langle R_{e e}(t)\right\rangle \sim t^{\nu}
$$

is $\nu \simeq 0.90(5)$. The inset in Fig. 1(a) displays the depen- dence of the measured $\nu$ on the network size $N$, which indicates that the values of $\nu$ approach to 1 as increasing $N$. This result agrees with the expectation on the SWN [16] when $p \rightarrow 1$. The $\left\langle R_{e e}(t)\right\rangle$ does not increase indefinitely, but rapidly reaches a saturation value $\left\langle R_{e e}(\bar{\ell}, t \rightarrow \infty)\right\rangle$ after a very short crossover time $\tau_{e e}$. Since $\tau_{e e}$ is nearly equal to 10 or slightly larger than 10 even for the very large network size ( or $N=10^{7}$ ), the expectation of $\nu=1$ from the data for nearly one decade of $t$ or so seems to be physically dangerous. However, since an earlier analytical study on the walks on a Cayley tree also suggests such $\nu=1$ behavior [23],$\nu \rightarrow 1$ as $N \rightarrow \infty$ is physically plausible one. We have also measured $\nu$ for the size upto $N=10^{7}$ and obtained the similar data as in Fig. 11(a). We thus expect that $\nu \rightarrow 1$ as $N \rightarrow \infty$ regardless of $\gamma$.

In Fig. 1(b) we display the $\left\langle R_{e e}(\bar{\ell}, t \rightarrow \infty)\right\rangle$ for $\gamma=4.3$ as a function of $\bar{\ell}$ with $\bar{\ell} \sim \ln N$. As shown in Fig. 1(b) $\left\langle R_{e e}(\bar{\ell}, t \rightarrow \infty)\right\rangle$ for $\gamma=4.3$ satisfies the power-law:

$$
\left\langle R_{e e}(\bar{\ell}, t \rightarrow \infty)\right\rangle \sim \bar{\ell}^{\alpha} .
$$

with $\alpha=0.93(2)$. $\left\langle R_{e e}(\bar{\ell}, t \rightarrow \infty)\right\rangle$ for various $\gamma$ is also found to satisfy the power law (5) very well. The obtained $\alpha$ 's for various $\gamma$ by assuming $\bar{\ell} \sim \ln \ln N$ for $\gamma<3$ and $\bar{\ell} \sim \ln N$ for $\gamma \geq 3$ are displayed in Table $\square$.

From the Eqs. (4) and (5), the dynamical scaling relation

$$
\left\langle R_{e e}(\bar{\ell}, t)\right\rangle=\bar{\ell}^{\alpha}(\gamma, N) g\left(t / \bar{\ell}^{z}\right) .
$$

is expected. The scaling function then $g(x)$ satisfies the relation

$$
g(x) \sim \begin{cases}x^{\nu} & , x \ll 1 \\ \text { const. } & , x \gg 1 .\end{cases}
$$

The dynamical scaling relation (6) also physically means that $\tau_{e e}$ scales as $\tau_{e e} \sim \bar{\ell}^{z}$. The dynamic exponent $z$ can thus be evaluated from the measured $\alpha$ and $z$ through the

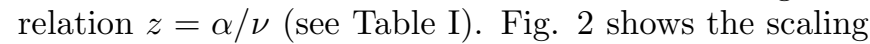
plot of $\left\langle R_{e e}\right\rangle$ measured on SFNs with $\gamma=2.4,3.0,4.3$ and on random networks (RNs) for $N=10^{3}, 10^{4}, 10^{5}$ and $10^{6}$ using Eq. (6). As shown in Fig. 2, $\left\langle R_{e e}\right\rangle$ 's for various $N$ collapse very well into a single scaling curve with the exponents listed in Table I for each network topology. Note that $\bar{\ell}$ in networks represents the average diameter by definition [1]. From the scaling collapse, we find the dependency of $\bar{\ell}$ on $\gamma$ and $N$ :

$$
\bar{\ell}(\gamma, N) \sim d \sim \begin{cases}\ln \ln N & , 2<\gamma<3 \\ \ln N & , \gamma \geq 3 .\end{cases}
$$

For SFN with $\gamma=3$, it is known that the diameter scales as $d \sim \ln N /(\ln \ln N)$ in the Barabási-Albert (BA) model [24] with looped structures [3, 25]. In contrast the BA network without looped structures or tree-type BA network satisfies $d \sim \ln N[3,25]$. For the best scaling collapse we use the relation $d \sim \ln N$ for $\gamma=3$ even though the static SFN 21] has loops (see Fig. 2(b)). To check the scaling behavior $d \sim \ln N$ for $\gamma=3$ obtained 


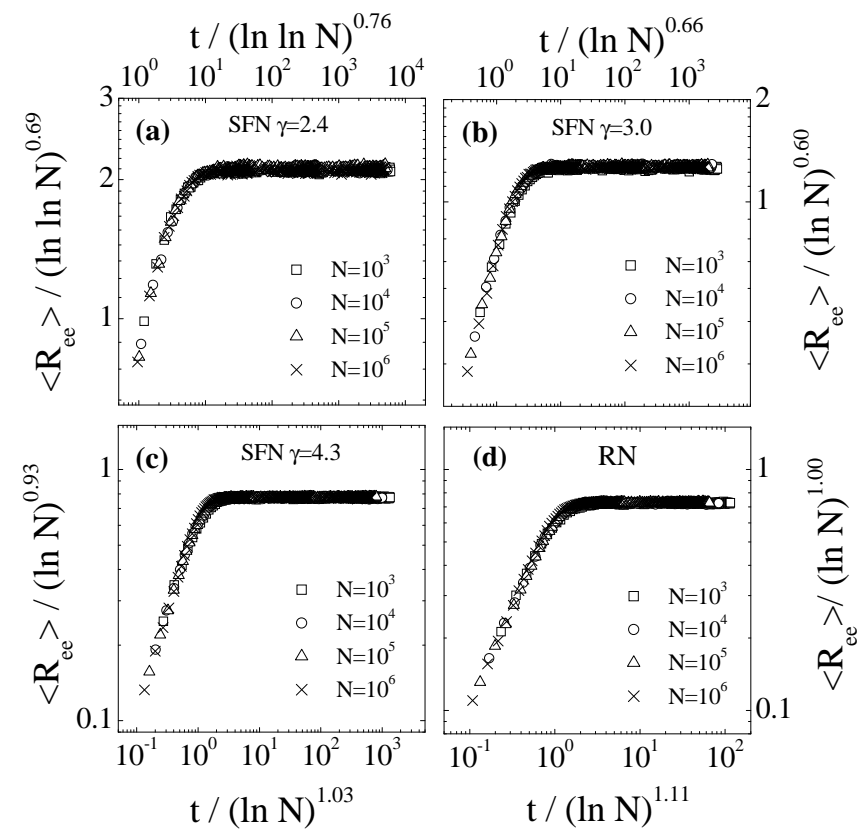

FIG. 2: Scaling plot of $\left\langle R_{e e}\right\rangle$ on SFNs with (a) $\gamma=2.4$, (b) 3.0 , (c) 4.3 and on (d) RNs for $N=10^{3}, 10^{4}, 10^{5}$ and $10^{6}$.
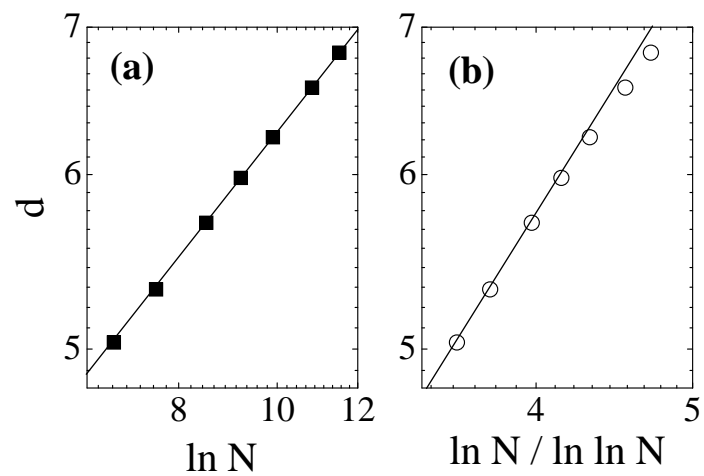

FIG. 3: Dependence of the diameter of static SFN with $\gamma=3$ on $N$. (a) The relation $d \sim \ln N$ is well satisfied. (b) The relation $d \sim \ln N / \ln \ln N$ deviates when $N$ increases.

by RW method, the diameter of SFN for $\gamma=3$ is directly measured by the BFA method (see Fig. 3). As can be seen from Fig. 3(a), the relation $d \sim \ln N$ is well satisfied for the static SFN with $\gamma=3$ and agrees with the result obtained by RW method or from Fig. 2] However, the relation $d \sim \ln N / \ln \ln N$ deviates when $N$ increases (Fig. 3(b)).

The results from Eqs. (6) and (7) provide another way to find the scaling behavior of $d$ in SFN. The result implies that the computing time needed for the measurement of scaling behavior of $d$ by RW method increases as $O\left((\ln N)^{z}\right)$ or $O\left((\ln \ln N)^{z}\right)$. Since the computing time to measure $d$ by BFA increases as $O\left(N^{2}\right)$, the RW method is far more efficient to find scaling behavior of $d$.

For comparison's sake we now explain the average number of distinct visited sites $N_{v}(t)$ on SFNs with var-
TABLE I: Estimates of the exponents $\alpha, z$ for SFNs with $\gamma$ and random network $(\mathrm{RN}) .(z=\alpha / \nu)$

\begin{tabular}{rrr|rrr}
\hline \hline$\gamma$ & $\alpha$ & $z$ & $\gamma$ & $\alpha$ & $z$ \\
\hline 2.15 & $0.42(1)$ & $0.46(3)$ & 3.0 & $0.60(1)$ & $0.66(4)$ \\
2.40 & $0.69(2)$ & $0.76(3)$ & 3.5 & $0.81(1)$ & $0.90(4)$ \\
2.50 & $0.84(1)$ & $0.93(4)$ & 4.3 & $0.93(2)$ & $1.03(5)$ \\
2.75 & $1.12(2)$ & $1.24(5)$ & 5.7 & $0.98(4)$ & $1.08(7)$ \\
2.90 & $1.49(1)$ & $1.65(6)$ & & & \\
\hline & & RN & $1.00(5)$ & $1.11(8)$ \\
\hline \hline
\end{tabular}

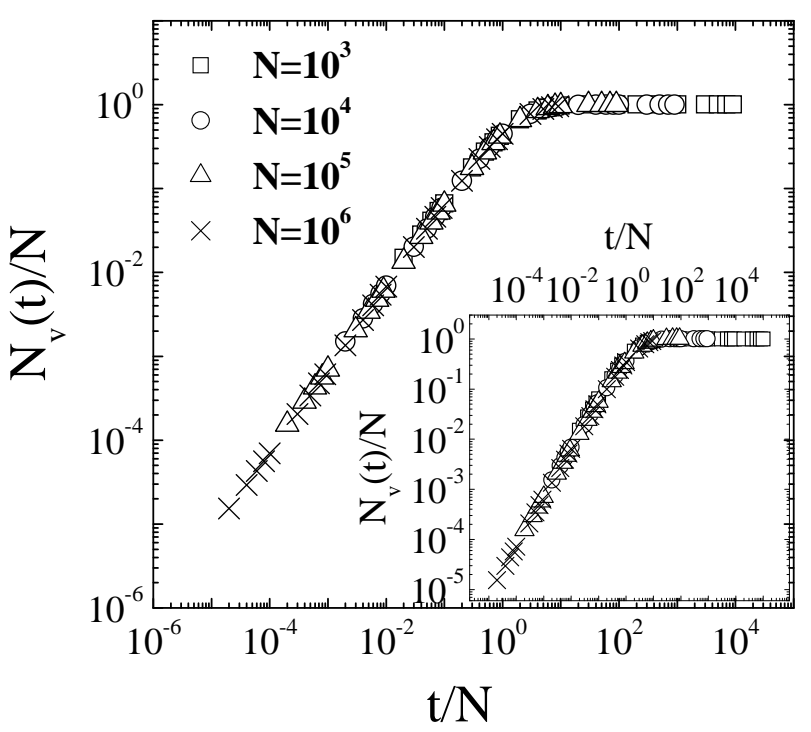

FIG. 4: This figure shows the scaling collapse for $N_{v}$ on SFN with $\gamma=4.3$ and 2.4 (inset).

ious $\gamma$. Fig. 4 4 shows the scaling plot of $N_{v}(t)$ for $\gamma=4.3$ and 2.4 against $t / N$ for $N=10^{3}, 10^{4}, 10^{5}$ and $10^{6} . N_{v}(t)$ on SFN satisfies the scaling relation

$$
N_{v}=N f(t / N)
$$

with

$$
f(x) \sim \begin{cases}x, & x \ll 1 \\ 1, & x \gg 1 .\end{cases}
$$

We have checked that the scaling relation (9) holds very well for SFNs with any $\gamma$.

The scaling relation (9) is slightly different from that of the regular lattice and the SWN. On the infinite $D$ dimensional lattices $N_{v}$ depends on $D$. In the limit of $t \rightarrow \infty$ [26], $N_{v} \sim \sqrt{t}$ in $D=1, N_{v} \sim t / \ln t$ in $D=2$ and $N_{v} \sim t$ for $D>2$. On SWNs, Almaas et el. 16 showed that $N_{v} \sim \sqrt{t}$ for $t \ll \xi^{2}$ (with $\xi=1 / p$ ), $N_{v} \sim t$ for $\xi^{2} \ll t \ll N \xi$ and $N_{v}=N$ for $t \gg N \xi$. This means that if the random walker does not reach the shortcut, then the walker always sees the regular lattice structure $(D=1)$. However, if the walker meets the shortcut, then the behavior of walker follows the mean-field result and finally $N_{v}$ saturates to $N$ due to the finite-size of the network. However, each node in SFN can be connected 
to any other nodes and SFN can be regarded as an infinite dimensional structure. Thus, $N_{v}$ on SFN follows the mean-field behavior after the first few steps. From Eqs. (9) and (10) the time $t_{\times}$at which $N_{v}=N$ is given by $t_{\times} \simeq N$. This implies that the walker can sample a new region at each time step by using shortcuts until it visits all nodes in the network. Thus the statistical properties such as the average of certain quantity can be significantly enhanced even for small RW steps.

Moreover, since $\left\langle R_{e e}(t)\right\rangle \sim t^{1 / 2}$ and $\left\langle N_{v}(t)\right\rangle \sim t$ for $D \geq 2$, we can obtain a relation between the $N_{v}$ and the number of accessible nodes (or volume) $N_{a c}$ within the radius $\left\langle R_{e e}(t)\right\rangle$ for $D \geq 2$ :

$$
\frac{N_{v}}{N_{a c}} \sim t^{1-D / 2} .
$$

Thus, $N_{a c} / N_{v}$ diverges as $t \rightarrow \infty$ which indicates the transient behavior of RWs [27]. The transient behavior becomes much more striking in the limit $D \rightarrow \infty$. From $\tau_{e e} \sim(\ln N)^{z}\left(\right.$ or $(\ln \ln N)^{z}$ and $t_{\times} \simeq N$, we know $\tau_{e e} \ll$ $t_{\times}$on SFN. This result means that the walker effectively moves to the end of SFN without visiting all nodes. As a result, we can measure the scaling behavior of $d(N)$ in much shorter time than BFA algorithm (for example, see Figs. 2 and 3 ).

We investigate the scaling properties of RWs on SFNs. We measure the end-to-end distance $R_{e e}$ and the number of distinct visited sites $N_{v}$. From the scaling ansatz for $\left\langle R_{e e}\right\rangle$ as Eq. (6), we have measured the scaling exponents $\alpha, \nu$ and $z$ for $\left\langle R_{e e}\right\rangle$ on various networks. From the scaling relation we find the dependency of $\bar{\ell}$ on $\gamma$ and $N$. Based on the simple scaling arguments, we also discuss the theoretical reasons why scaling behavior of $d(N)$ of networks can be measured far more efficiently by RW method than by BFA.

We thank to Dr. Kwon and Dr. Yoon for useful discussions. This work is supported by grant No. R012006-000-10470-0 from the Basic Research Program of the Korea Science \& Engineering Foundation.
[1] S. N. Dorogovtsev and J. F. F. Mendes, Adv. Phys. 51, 1079 (2002); R. Albert and A.-L. Barabási, Rev. Mod. Phys. 74, 47 (2002).

[2] H. Jeong, B. Tombor, R. Albert, Z. N. Oltvai, and A.L. Barabási, Nature 407, 651 (2001); S.-H. Yook Z. N. Oltvai, and A.-L. Barabási, Porteomics 4, 928 (2004).

[3] S. Bornholdt and H. G. Schuster, Handbook of Graphs and Networks (Wiley, New Yok, 2002).

[4] S. N. Dprogovtsev and J. F. F. Mendes, Evolution of Networks (Oxford University Press, Cambridge, UK, 2003).

[5] S. H. Strogatz, Nature (London) 410, 268 (2001).

[6] B. Bollobás, Random Graphs (Academic, London, 1985).

[7] E. Ben-Naim, P. L. Krapivsky, and S. N. Majumdar, Phys. Rev. E 64, R035101 (2001).

[8] P. Erdös and A. Rényi, Publ. Math. 6, 290 (1959).

[9] D. J. Watts and S. H. Strogatz, Nature (London) 393, 440 (1998).

[10] R. Cohen and S. Havlin, Phys. Rev. Lett. 90, 058701 (2003).

[11] R. Sedgwick, Algorithms (Addison-Wesley, 1988); M. E. J. Newman, Phys. Rev. E. 64, 016132 (2001).

[12] A. V. Goltsev, S. N. Dorogovtsev and J. F. F. Mendes, Phys. Rev. E 67, 026123 (2003).

[13] R. Cohen, D. ben-Avraham and S. Havlin, Phys. Rev. E 66, 036113 (2002).

[14] J. D. Noh and H. Rieger, Phys. Rev. Lett. 92, 118701
(2004)

[15] J. D. Noh and S.-W. Kim, J. Korean Phys. Soc. 48, S202 (2006).

[16] E. Almaas, R. V. Kulkarni, and D. Stroud, Phys. Rev. E 68, 056105 (2003).

[17] S. N. Dorogovtsev, A. V. Goltsev and J. F. F. Mendes, Phys. Rev. Lett. 66, 016104 (2002).

[18] S. Lee and Y. Kim, Phys. Rev. E 71, 057102 (2005).

[19] S. Kwon, S. Lee and Y. Kim, Phys. Rev. E 73, 056102 (2006).

[20] S. Lee, S. -H. Yook and Y. Kim, Phys. Rev. E 74, 046118 (2006).

[21] K.-I. Goh, B. Kahng and D. Kim, Phys. Rev. Lett. 87, 278701 (2001).

[22] M. N. Barber and B. W. Ninham, Random and Restricted Walks (Gordon and Breach, New York, 1970).

[23] Y. Kim, Phys. Rev. A 39, 4923 (1989).

[24] A.-L. Barabási and R. Albert, Science 286, 509 (1999).

[25] G. Szabó, M. Alava, and J. Kertész, Phys. Rev. E 66, 026101 (2002).

[26] A. Dvoretzky and P. P. Erdös, in Proceedings of the 2nd Berkeley Symposium on Mathematical Statistics and Probability (University of California Press, Berkeley, California, 1950), pp. 353-367.

[27] G. Pólya, Mathematische Annalen 84, 149 (1921). 